Itinéraires Itinéraires

Littérature, textes, cultures

2012-3 | 2013

Lire les villes marocaines

\title{
Priska Degras, L'Obsession du Nom dans le roman des Amériques
}

Paris, Karthala, 2011, 264 pages.

\section{Annick Gendre}

\section{OpenEdition}

\section{Journals}

Édition électronique

URL : http://journals.openedition.org/itineraires/1032

DOI : 10.4000/itineraires. 1032

ISSN : 2427-920X

\section{Éditeur}

Pléiade

\section{Édition imprimée}

Date de publication : 1 juillet 2013

Pagination : 181-185

ISBN : 978-2-343-01183-7

ISSN : 2100-1340

\section{Référence électronique}

Annick Gendre, «Priska Degras, L'Obsession du Nom dans le roman des Amériques », Itinéraires [En ligne] 2012-3 | 2013, mis en ligne le 01 décembre 2012, consulté le 22 septembre 2020. URL : http:// journals.openedition.org/itineraires/1032 ; DOI : https://doi.org/10.4000/itineraires.1032

Ce document a été généré automatiquement le 22 septembre 2020.

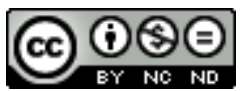

Itinéraires est mis à disposition selon les termes de la licence Creative Commons Attribution - Pas d'Utilisation Commerciale - Pas de Modification 4.0 International. 


\section{Priska Degras, L'Obsession du Nom dans le roman des Amériques}

Paris, Karthala, 2011, 264 pages.

Annick Gendre

\section{RÉFÉRENCE}

Priska Degras, L'Obsession du Nom dans le roman des Amériques, Paris, Karthala, 2011, 264 pages.

«Les non-dupes errent.»

Jacques Lacan

L'Obsession du Nom dans le roman des Amériques que Priska Degras a publié en 2011 aux éditions Karthala est un essai qui fera date. Non seulement il propose une synthèse sur une question, celle de la praxis attachée au nom propre de personne ou « Nom», dans les archipels, question qui intéresse les historiens et les linguistes depuis les années $1980^{1}$, mais ses pages s'emparent également de la problématique de la nomination dans le «roman des Amériques». Le terme roman y est envisagé au-delà de sa seule acception générique, dans le sens de construction imaginaire partagée, « commune ».

2 Le corpus choisi revisite ainsi l'appellation initiée par Édouard Glissant dans le chapitre intitulé «Le Roman des Amériques », extrait de sa thèse publiée sous le titre Le Discours antillais (1981) : au-delà de l'articulation des îles et du continent américains, «des sociétés qui connurent l'esclavage et le système des plantations » (p. 6), on reconnaît les questionnements majeurs que signalait cette expression pour l'écrivain martiniquais, tels que la "hantise du passé ", la «quête de la durée » et la "mémoire du futur», la "parole du paysage», "l'irruption dans la modernité [...] hors la continuité littéraire » et la " liaison très tourmentée entre écriture et oralité ${ }^{~}$ ». Ainsi la réflexion de Priska Degras réunit-elle des écrits composés dans les trois langues anciennement "impériales», traversées par d'autres langues, sans omettre d'interroger leurs traductions en français. Privilégiant une écriture et précédé d'un 
exergue qui scande subtilement le parcours, chaque chapitre engage une nouvelle étape de la réflexion, si bien que l'ordre chronologique n'est nullement de rigueur.

3 Au seuil de son essai, l'auteure indique les spécificités de la question du Nom au sein du corpus choisi : la métaphorisation de l'Histoire par le Nom, dans le prisme historique que constituent l'« esclavage » et le "système des plantations ». Dans l'accès, accordé comme empêché, au Nom, le caractère déterminant de l'institutionnalisation apparaît dès le premier chapitre, grâce au rappel de l'ambiguïté des pratiques de nomination autour de l'abolition de 1848. L'enjeu symbolique du Nom dans le contexte colonial et abolitionniste (l'annulation du Nom, comme « signe premier » d'asservissement et « de déshumanisation » (p. 14), « l'illégitimité de sa présence au monde [celle de l'esclave] », ainsi que l'ambivalence et les résistances dans les attributions massives du Nom autour de 1848) est mis en perspective avec les possibilités d'habiter ou non le Nom « attribué, et non transmis » (p. 32).

4 En effet, l'attribution d'un prénom pour patronyme aux nouveaux affranchis est source d'ambivalence : d'une part, accordé à l'individu libre, le prénom-patronyme largement utilisé exhibe l'ancien état d'asservissement; d'autre part, il atteste, dans la reconnaissance du passage de ce statut à la liberté, l'introduction du fantasme de l'enfant trouvé : en effet, en France, il était d'usage de donner pour patronyme aux orphelins le nom du saint du jour de leur naissance s'il était connu, sinon du jour où ils avaient été trouvés. Le prénom-patronyme, «les sobriquets ridicules, les noms de personnages historiques» (p. 22), les homophonies dans les nominations de masse, le choix du nom de l'ancien maître en en inversant ou non les syllabes, le projet de dénombrement, pour ne retenir que ceux-ci, jusqu'aux rapports rendant compte de la peur que se franchisse grâce à la nomination « la ligne de couleur par les libres » (p. 21) sont autant de pratiques qui prouvent combien l'attribution du Nom et son écriture dans les actes ont été prétexte à l'institutionnalisation de l'irrationnel présent dans le fait colonial. Sans jamais céder au jugement de valeur, Priska Degras montre comment s'est (re)jouée "dans cette obsession du nom, [...] l'image condensée, le résumé hautement signifiant des rapports raciaux dans la colonie» (p.24). Cette praxis de nomination agressive a généré plusieurs stratégies de résistances à la surdétermination du Nom, et simultanément un manque qu'explorent «nombre de productions littéraires des Amériques». Ce manque semble autoriser en effet "une lecture de l'Histoire, [...] celle de la filiation et de son déni» (p. 35), ainsi que représenter «le territoire neuf d'une liberté sans limite » (p. 36).

Passé le seuil de l'essai, neuf chapitres consacrés à neuf écritures distinctes des Amériques sont envisagées successivement.

5 L'enjeu premier du Nom est l'accès au langage, de pouvoir engager autant que répondre à « la tentative obstinée de renommer un monde, une communauté, et de faire de son histoire un récit signifiant» (p. 38). Le "premier innommable qu'est l'esclavage » lui oppose une béance nodale, selon Édouard Glissant. La fiction représenterait un moyen de dépasser cet innommable, thèse que partage l'auteure de l'essai. Si Pluie et vent sur Télumée miracle, de Simone Swartz-Bart permet de confirmer la récurrence des thèmes de «l'orphelinat et [de] la déréliction » (Jean Bernabé), l'analyse de Priska Degras pose les jalons de nuances précieuses pour la compréhension en contexte du corpus donné : par exemple, l'absence de Noms ne coïncide pas forcément avec une disparition du père, ni par conséquent avec une absence d'imago paternel ; le Nom absent n'implique pas un manque de cohésion dans la structure familiale. C'est aussi rappeler que les 
enjeux symboliques de la nomination peuvent emprunter d'autres voies et ne sont pas réductibles au signifiant du Nom reçu. D'où l'attention accordée au "refus de nommer ", en tant que "refus de la parodie du nom - telle qu'elle s'effectua à la libération de 1848 - l'exercice d'un pouvoir autonome de la communauté » (p. 76). Ce sont moins les stratégies contre la surdétermination du Nom, que l'investissement progressif du processus de nomination par le sujet ou par une communauté, qui sont retracés ici.

6 Ce pouvoir sur le Nom autorise la réflexion à délaisser le Nom du personnage dans le récit au profit du Nom de l'auteur, avec Saint John Perse. Le poète diplomate a très tôt décidé de signer ses textes d'un Nom littéraire qu'il faisait dériver de son Nom hérité. Cette nomination choisie manifeste une relation entre le mode de présence au monde et au paysage avec l'appréhension du Nom par le sujet qui le porte. La mise en scène du Nom, comme mode d'être à l'espace, croise l'investissement du Nom tel que le décline Toni Morrisson, en particulier dans La Chanson de Salomon : «la question des noms des personnes et des lieux lui [à la question du langage] est, évidemment, étroitement liée comme elle est liée [...] à celle de la légitimité et à celle de la faute, de la transgression » (p. 96). L'histoire secrète des Noms, chère à l'écrivaine, trouve un prolongement dans l'invisibilité et l'anonymat déclinés par le récit Homme invisible, pour qui chantes-tu? de Ralph Ellison. L'« indispensable voix sans nom» (p. 136) que finit par représenter le personnage principal se meut en une voix qui parle pour, au nom de.

7 C'est précisément une telle substitution qu'a refusée l'écrivain Naipaul, en rejetant le «nom commun [partagé] Antillais ». Il s'agit « d'établir le territoire de l'écriture hors de toute limite assignée, qu'elle soit celle de l'origine, de la "couleur" ou de l'Histoire » (p. 147). Idéologiquement et historiquement marquée, la réflexion de René Ménil n'en critique pas moins, au sein de la pensée de la négritude, les écueils de l'assignation par la nomination et met en garde contre l'« exotisme intérieur ». Priska Degras cite ce très beau commentaire de Ménil, reprenant Borges: «Mahomet, comme Arabe, était tranquille : il savait qu'il pouvait être arabe sans chameaux » (p. 157). Un autre pas est encore franchi vers la non-assignation, grâce au nom propre collectif de la figure du «Rebelle " chère à Aimé Césaire. Plus que d'un choix, il s'agit de décider de «ne pas faire de la période coloniale le surdéterminant obligatoire de tous les peuples extraeuropéens» (p.163) et de "garder l'initiative historique» (p.167). Le principe d'« hétérogénéité ", qui préside à la fondation des grands ensembles culturels doit relever d'un processus intérieur, non d'une volonté extérieure. Dans ses deux pièces de théâtre, La Tragédie du roi Christophe et Une Saison au Congo, Césaire revisite l'histoire de la création de l'État-nation, à l'aube de la décolonisation. Christophe et Lumumba se heurtent, chacun à leur façon, à la difficulté de «nommer ce qui n'a jamais » encore « existé » (p. 172). À ces deux figures emblématiques du « rebelle » répond la figure du dictateur tropical, telle qu'elle apparaît dans L'Automne du Patriarche de Gabriel García Márquez et dans Moi, Trésilien-Théodore Augustin de Xavier Orville. Tandis que le premier sombre dans un engloutissement " de l'usure du temps », l'autre projette l'instauration d'un temps neuf, soit « une nouvelle aube de l'Histoire » (p. 193). Ainsi représentent-ils tous deux «le visage multiple et unique du manque de l'Histoire» (p. 197). Dans ce parcours qui relie le Nom au manque de l'Histoire, l'archipélisation qu'illustre Ormerod d'Édouard Glissant introduit, avec le peuple fictionnel des Batoutos, «la pensée de la trace et de l'errance », également déclinées par des penseurs relevant d'autres régions du monde ${ }^{3}$. 
8 Les deux chapitres liminaires explorent deux types de fading du Nom, la démultiplication du Nom et la vacance nominative. L'hétéronomie de Fernando Pessoa et les identités exclusives qu'interroge Milan Kundera placent la question de la multiplication du Nom de l'écrivain sous le signe des limites de l'identité, tandis que les personnages Coleman Silk de Philip Roth (La Tache) et Theolonius Monk Ellison de Percival Everett (Effacement) font l'apprentissage du «droit de choisir son appartenance » (p.225). À l'inverse, le personnage d'Egudu, de Coetzee (Elisabeth Costello) revendique sa "fidélité à son essence » (p. 231), choisissant ainsi le " nous » plutôt que le « je ». Ce sont autant de positionnements quant au Nom qui interrogent la liberté du sujet. Notons que le choix d'articuler les Noms de personne écrivains avec les Noms dans le texte littéraire enrichit considérablement l'analyse. Le rêve mexicain d'Antonin Artaud et la forêt d'Alejo Carpentier, comme celle de Wilson Harris, interrogent la dilution de la nomination dans l'immensité de l'espace américain, vacance nominative qui signifie aussi possibilité d'un nouveau récit du monde.

Le parcours de cette réflexion s'achève en convoquant Apex ou Le Cache-blessure de Colson Whitehead, dont le personnage principal a pour profession la création de noms commerciaux. Cristallisant ainsi plusieurs positionnements et stratégies nominatives, le récit éprouve alors non seulement le Nom en tant que masque, mais l'interroge aussi en tant que «mot juste » (Carlos Fuentes). La richesse représentative du corpus choisi et la générosité de cette pensée du Nom que nous offre Priska Degras fait de cet essai un ouvrage rare pour son éclairage rigoureux sur le foisonnement de "l'énigme du Nom » (Jacques Lacan), qui fait trace de l'usage libre de soi par soi.

\section{NOTES}

1. Voir notamment la thèse soutenue en 1983 de Sudel Fuma, La Mémoire du nom ou " Le nom, image de l'homme ", l'histoire des noms réunionnais à partir des registres d'affranchis de 1848 (t. I et II), ANSOM, bibliothèque AOM TH/869 et les travaux de Jean Bernabé, cités par Priska Degras.

2. Édouard Glissant, Le Discours antillais [1981], Paris, Gallimard, 2002, p. 435-443.

3. Voir, à cet effet, L'Écriture et la différence, Demeure Athènes, Khôra, de Jacques Derrida ; La Mémoire, l'histoire, l'oubli, de Paul Ricœur, les œuvres de Edmond Jabès et de Abdelkébir Khatibi.

\section{AUTEURS}

\section{ANNICK GENDRE}

Université d'Orléans, POLEN/CEPOC 